\title{
Quantifying vasculature: new measures applied to arterial trees in the quail chorioallantoic membrane
}

\author{
SHARON R. LUBKIN*†, SARAH E. FUNK $\$$ and E. HELENE SAGE $\$$
}

$\dagger$ Department of Mathematics, North Carolina State University, Raleigh, NC 27695-8205, USA

$\ddagger$ Hope Heart Program, The Benaroya Institute at Virginia Mason, 1201 Ninth Avenue, Seattle, WA 98101, USA

(Received 8 March 2004; revised 25 January 2005; in final form 29 June 2005)

\begin{abstract}
A wide variety of measures is currently in use in the morphometry of vascular systems. We introduce two additional classes of measures based on erosions and dilations of the image. Each measure has a clear biological interpretation in terms of the measured structures and their function. The measures are illustrated on images of the arterial tree of the quail chorioallantoic membrane (CAM). The new measures are correlated with widely-used measures, such as fractal dimension, but allow a clearer biological interpretation. To distinguish one CAM arterial tree from another, we propose reporting just three independent, uncorrelated numbers: (i) the fraction of tissue which is vascular $\left(V F_{0}\right.$, a pure ratio), (ii) a measure of the typical distance of the vascularized tissue to its vessels ( $C L$, a length), and (iii) the flow capacity of the tissue ( $P$, an area). An unusually large $C L$ would indicate the presence of large avascular areas, a characteristic feature of tumor tissue. $C L$ is inversely highly correlated with fractal dimension of the skeletonized image, but has a more direct biological interpretation.
\end{abstract}

Keywords: Vascular; Fractal; Chorioallantoic membrane; Angiogenesis; Cancer

\section{Introduction}

How does one count blood vessels? For small numbers, we begin with "one, two, three...." Over larger areas, we commonly think of some measure of vascular density, the number of vessels per unit area or volume (as in, e.g. [10]). There is a tremendous variety of measures in use (for an overview, see [9]), and many of them are not necessarily intuitive. For example, if we care primarily about vessel length, we should use a measure of length density. Length density is computed from a skeletonized image. If we prefer ease of measurement, we use area density, the fraction of a $2 \mathrm{D}$ image which is occupied by vessels and their lumens. Area density corresponds to volume fraction, the fraction of voxels in a 3D image which are occupied by vasculature.

Choosing the right measure of vascular density requires clarifying the purpose of the measurement. If our primary interest is in how much mass of vascular tissue is present, then volume fraction is the correct measure-but we must exclude the lumens. If we are instead interested in the total volume of the vasculature and its contents, we use volume fraction and include the lumens. However, often the feature that we are investigating is not anatomical but functional: in the case of vascularization our underlying concern is the flow. With the correctly derived measure, we can estimate flow capacity from an image.

Fractals have become a very popular metric for vascular systems e.g. [5], and software for computing the fractal dimension of an image has become fairly widespread. For many systems, the arterial tree is very well represented by a fractal. The fractal dimension is a unitless number, which can be tracked over time and compared across treatments. It is straightforward to compute, but it does come with some statistical liabilities [2].

We know what fractal dimension represents mathematically, but what it signifies biologically is less clear. We do know that fractal dimension of the chorioallantoic membrane (CAM) increases during development $[4,6,11]$. That mathematical fact, however, does not tell us which biological quantity is increasing over time, for which the fractal dimension is an indicator. Is it total flow? Is it flow homogeneity? We know that tumor vasculature has a higher fractal dimension than normal vasculature, and increases over time (for review, see [1]), yet a low fractal dimension can be associated with a high grade of tumor and poor patient outcome [8]. What is the biological interpretation of these observations? Figure 1 illustrates two CAM arterial trees with the same fractal dimension, yet there are obvious differences in the structures.

*Corresponding author. Email: lubkin@eos.ncsu.edu

Journal of Theoretical Medicine

ISSN 1027-3662 print/ISSN 1607-8578 online (c) 2005 Taylor \& Francis

http://www.tandf.co.uk/journals

DOI: $10.1080 / 10273660500264684$ 

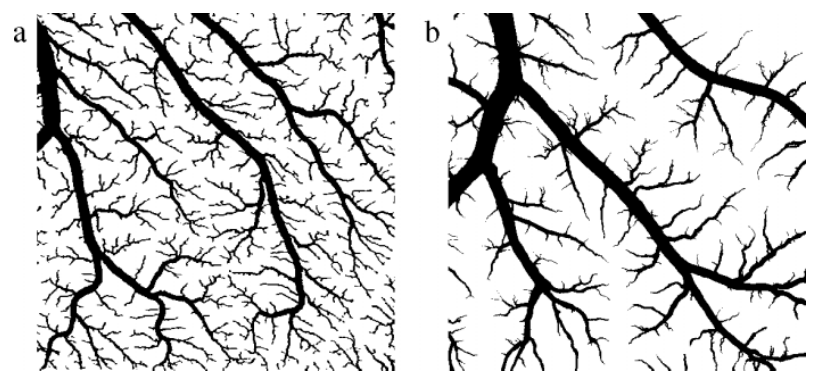

Figure 1. Images from two CAM arterial trees. Scale is identical. Fractal dimension is the same (a) $D_{f}=1.54 \pm 0.04$, (b) $D_{f}=1.53 \pm 0.02$ yet there are major differences between the two trees.

The fractal dimension alone cannot explain the difference, which is obvious to the eye. The eye sees that there must also be significant differences in the function of these two trees.

The goal of this paper is to present a discussion of the issues of vascular quantification and to suggest additional measures of a vascular tree. We illustrate the measures using a set of images of the CAM arterial tree and discuss their biological significance.

\section{Methods}

Fertilized quail eggs were cultured and imaged at 72 pixels/in as in Parsons-Wingerter et al. [6]. Background and veins were erased by hand, arteries were filled by hand, and images were binarized, using NIH Image. Thresholds for binarization were determined by manually adjusting to retain the smallest arterial branches visible [6]. Skeletonization of binary images was done by NIH Image (figure 2). Image analysis was done on binary.tif files in Matlab.

\subsection{Definitions of measures}

The two most commonly used measures in the vascular morphometry literature go by almost as many names as the number of papers in which they appear. We will call them the vascular fraction and the fractal dimension of the skeletonized tree.

\subsection{Vascular fraction}

This is the fraction of the tissue volume (in 3D) or area (of a $2 \mathrm{D}$ slice) which is occupied by the vessels. It is the

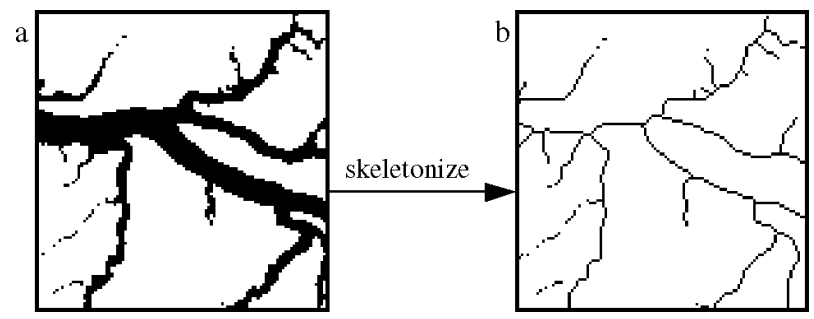

Figure 2. CAM image (a) is skeletonized by removing boundary pixels until the remaining object (b) is only 1 pixel thick. most obvious and straightforward measure of the gross amount of vasculature in a tissue. In this study, vascular fraction $V F$ counts only the arterial tree. It is unitless, and in our sample ranges from $15-20 \%$. It is computed for a 2D structure by counting vascular pixels in the 2D binary image, as a fraction of the total pixel number. The vascular fraction of a 3D structure can be determined either by voxel vascular fraction in a 3D stack, or extrapolated from pixel vascular fraction of a 2D slice. There are issues relating to the proper thresholding in the image analysis, and these can be exacerbated by heterogeneity in marker uptake $[3,10]$, but vascular fraction remains conceptually the most robust measure of vascular tissue.

\subsection{Fractal dimension}

The fractal dimension is a unitless quantity. It can be defined as the negative of the slope of the $\log -\log$ plot of the number of pixels in the vascular portion against the size of those pixels. As in $[4,6]$, we use the box-counting method: for each of several box sizes, the image is divided into a grid, and boxes that contain some vessel are counted. The slope of the $\log -\log$ graph is computed by linear regression. We define $D_{f}$ as the fractal dimension of the arterial tree, and $D_{f s k}$ as the fractal dimension of the skeletonized tree. They are different measures. Although $D_{f s k}$ is far more commonly used in the vascular morphometry literature than is $D_{f}$, it is most often referred to simply as the fractal dimension. In this paper, we make a clear distinction between $D_{f}$ and $D_{f s k}$, because they are correlated with different biological quantities. Our method for calculating $D_{f}$ was tested on several artificial fractal images (gif) whose $D_{f}$ is known from mathematical

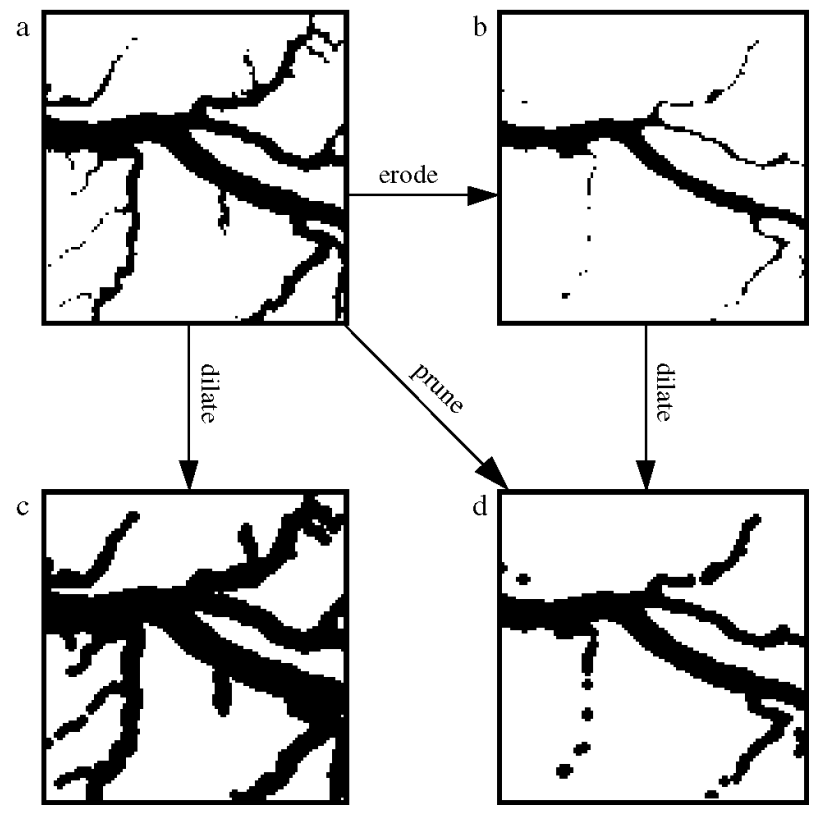

Figure 3. CAM image (a) modified by (b) erosion, (c) dilation, and (d) pruning processes. Pruning is accomplished by erosion followed by dilation. In each case, the gauge is a disk of radius 4 pixels. Hence pruning by a gauge of 4 pixels digitally removes all structures of radius smaller than 4 pixels, while preserving the size of all thicker structures. 
principles (Koch snowflake and Sierpinski triangle, square, pentagon and hexagon). Our algorithm slightly underestimates $D_{f}$ for the test images by a mean of 0.05 . When this factor is applied to correct the measurements, the error in $D_{f}$ as compared to the true (mathematically derived) $D_{f}$ is within 0.02 for all test images. We do not include this correction factor in our reported $D_{f}$ or $D_{f s k}$ for the natural (CAM) images.

\subsection{Erosion and dilation}

We examined a set of dilations and prunings of the vascular tree. A dilation of the tree is the region in the image which is within a particular distance of the vessels (figure 3). The complement of the dilation is the fraction of the tissue which is at least a particular distance from the vessels. The complement farthest from the vessels is, for example, more hypoxic. Finding the vascular complement fractions $C F(r)$ at all possible distances $r$ from the vasculature (figure 4) allows us to compute measures of perfusion efficiency, such as the median distance to a vessel, or to locate regions in the tissue which are unusually far from a vessel (figure 5).

A pruning of the vascular tree is a subset of the vascular tree which has all its branches below a certain radius trimmed (figure 6). The set of vascular fractions $V F(r)$ of all possible pruning radii $r$ could allow us to make measurements of the flow efficiency, for example. Pruning is accomplished by first eroding the image by a disk of radius $r$, then dilating by the same amount (figure 3 ).

\subsection{Regression}

The measured functions $V F(r)$ and $C F(r)$ can be approximated by curves, using nonlinear regression, or linear regression on transformed data. We thus determine parameters tuning these curves, which distinguish between vascular trees with different anatomical features. In any regression, it is important not simply to have small residuals $\left(R^{2}\right.$ large) but also to observe no trend in the residuals.

\section{Results}

\subsection{Fractal measures}

The CAM images that we analysed (displayed in figure 11) were all very well-described by fractals $D_{f}$ and $D_{f s k}$.

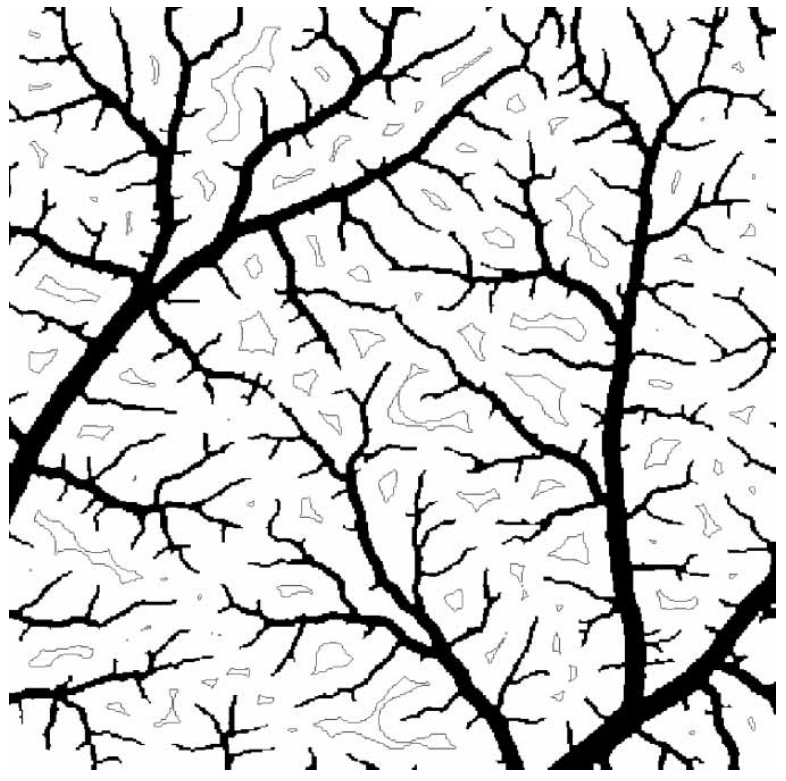

Figure 5. Significance of complement fraction. Outlined in image is the portion of the tissue which is in the upper 5\% of distance to a vessel. The 95th percentile distance is $325 \mu \mathrm{m}$; hence we say that $95 \%$ of the tissue is within $1 / 3 \mathrm{~mm}$ of a vessel, and $C F(325 \mu \mathrm{m})=0.05$. The spatial distribution of these poorly vascularized regions is fairly uniform in the CAM; however, in tumor tissue we would expect much more patchiness.

The curves of pixel size against vessel count were highly linear for $D_{f}\left(R^{2}>0.998\right)$ and $D_{f s k}\left(R^{2}>0.97\right)$, though for $D_{f s k}$ there was a slight trend in the residuals, a slight downward curvature at the coarser scales. Hence, we may reasonably assume that the CAM arterial tree is fractal rather than multifractal [13]. The range of fractal dimensions was quite small in our collection of CAM images. $D_{f}$ ranged from $1.43 \pm 0.02$ to $1.54 \pm 0.04$. $D_{f s k}$ ranged from $1.02 \pm 0.05$ to $1.13 \pm 0.10$. Note that both measures had an observed range in the CAM of 0.11 , but $D_{f s k}$ had a much larger standard error of each individual measurement.

\subsection{Vascular fractions}

The vascular fractions of the prunings, $V F(r)$, were modelled by several curves, in particular, a linear, an exponential, a power law, a Weibull function, and a quadratic function. Surprisingly, all these function families had either poor fits or strong trends in the residuals, except for two functions.

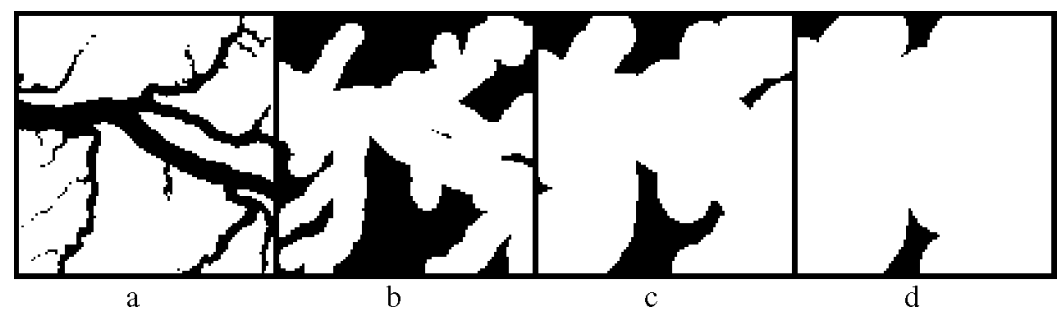

Figure 4. Calculation of complement fraction $C F(r)$. Original image (a) and regions of the tissue which are at least (b) 10, (c) 20, and (d) 30 pixels from any vessel. Complement fractions $C F(r)$ : (a) $78 \%$, (b) $38 \%$, (c) $15 \%$, (d) $5 \%$. 

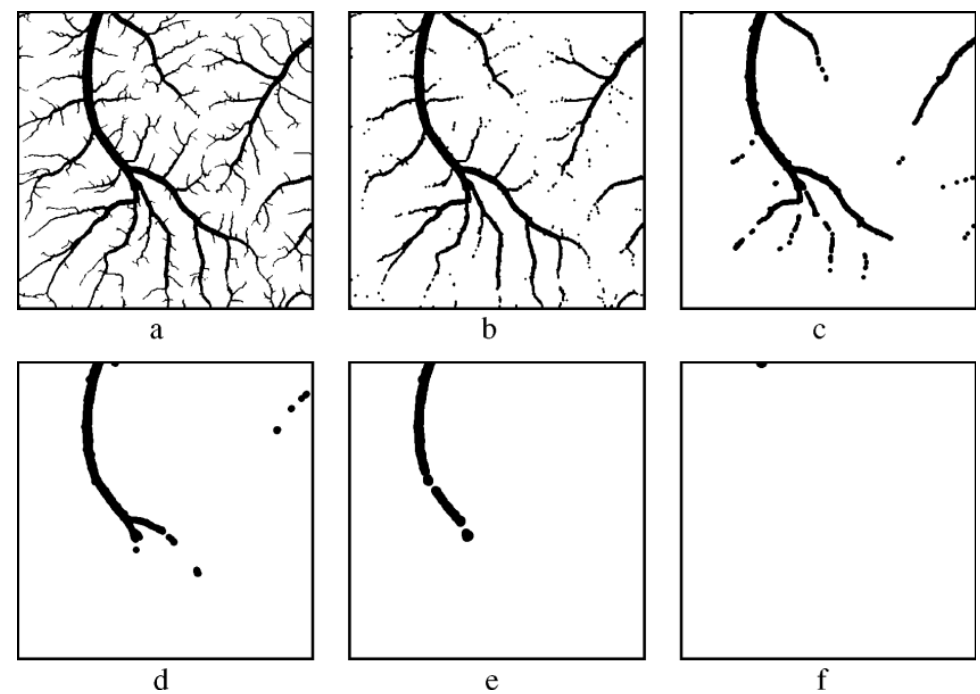

Figure 6. Prunings of a CAM arterial tree at different radii: (a) original arterial tree, (b-e) all vessels of radius below the gauges (b) $48 \mu \mathrm{m}$, (c) $96 \mu \mathrm{m}$, (d) $144 \mu \mathrm{m}$, (e) $192 \mu \mathrm{m}$, (f) $240 \mu \mathrm{m}$ have been digitally pruned (removed). Note that digitally-pruned vessels may not remain connected. Vascular fraction $V F(r)$ can be determined from this process as a function of pruning radius $r . V F(0 \mu \mathrm{m})=15.4 \%, V F(48 \mu \mathrm{m})=11.4 \%, V F(96 \mu \mathrm{m})=6.2 \%$, $V F(144 \mu \mathrm{m})=3.1 \%, V F(192 \mu \mathrm{m})=2.2 \%, V F(240 \mu \mathrm{m})=0.0 \%$.

One was the quadratic function $V F_{q}(r)=V F_{0}[1-$ $\left.\left(r / L_{q}\right)\right]^{2}$ where $r$ and $L_{q}$ are in $\mu \mathrm{m}$ or $\mathrm{mm}$ and $V F_{O}$ is unitless. Regression was linear on transformed data, fixing $V F_{O}$ at the measured value, with $R^{2}>0.93$. Range of $L_{q}$ was $233-581 \mu \mathrm{m}$ with SE $8-37 \mu \mathrm{m}$.

The other function which performed well was a compound exponential, $V F_{e}(r)=\exp \left(\ln \left(V F_{0}\right) \exp \left(r / L_{e}\right)\right)$, fitting $V F_{O}$ and $L_{e}$ for each curve. The range of $L_{e}$ was $158-421 \mu \mathrm{m}$, with SE $8-28 \mu \mathrm{m}$, and $R^{2}>0.86$. Interestingly, the fitted values of $V F_{O}$ for the compound exponential model differed from the observed (raw) $V F_{0}$, with a correlation of only 0.72 . The compound exponential fit least well at $r=0$, where the data for $V F(r)$ were unusually level (figure 7). This may be an artifact of the imaging method, or it may be an artifact of the vascular growth process itself. It is reasonable to assume that there is a minimum size of capillary below which we see no vessels at all; this would naturally tend to level off the curve of $V F(r)$ near $r=0$, as we observe.

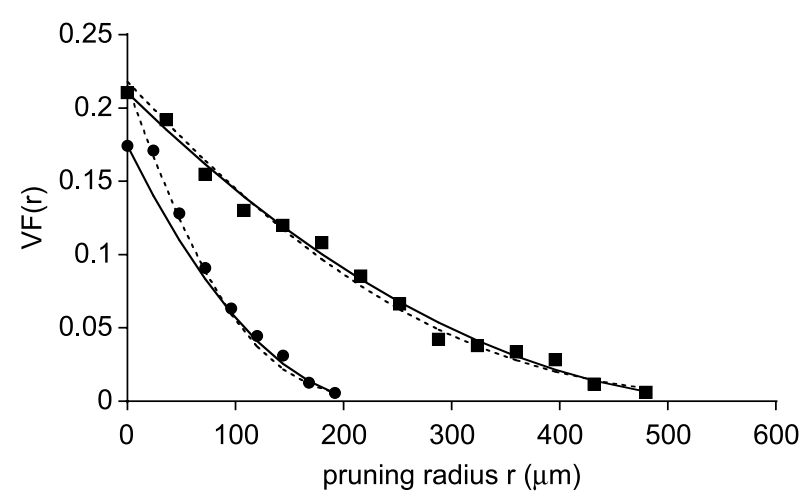

Figure 7. Vascular fraction $V F(r)$ after prunings to radius $r$. Shown are $V F(r)$ for the images with $L_{e}$ and $L_{q}$ the smallest $\left(L_{e}=158 \pm 9 \mu \mathrm{m}\right.$, $\left.L_{q}=233 \pm 8 \mu \mathrm{m}\right)$ and largest $\left(L_{e}=421 \pm 16 \mu \mathrm{m}, L_{q}=581 \pm\right.$ $17 \mu \mathrm{m}$ ). Fitted curves are $V F_{e}(r)$ (dashed) and $V F_{q}(r)$ (solid).
The $R^{2}$ values are fairly low, but that is because the data for $V F(r)$ have natural irregularity (figure 7).

\subsection{Complement fractions}

The complement fraction, $C F(r)$ (see figure 8), was fitted to several test functions, including an exponential decay and several hyperbolas, but the only functional form examined which had an excellent fit to all images and had no trend in the residuals was $C F(r)=$ $C F_{0} \exp (1-\exp (r / C L))$, where $C L$ is a characteristic length $(\mu \mathrm{m})$ and $C F_{O}$ is the highest value of $C F$ (the same as $\left.1-V F_{0}\right)$. For this function, all images had $R^{2}$ $>0.99$.

Table 1 presents the measurements for a sample of 8 images. Standard errors are reported for all quantities except $V F_{O}$ and $C F_{0}$, which are dependent solely on the pixel size, which was small enough that we assume negligible error.

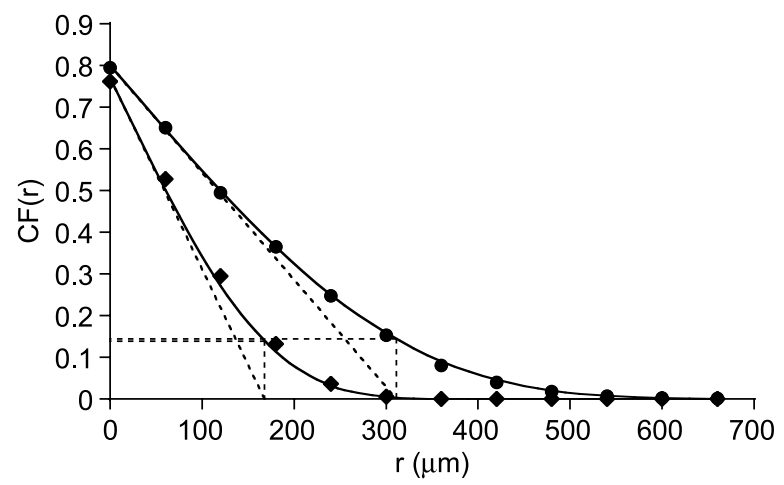

Figure 8. Complement fraction $C F(r)$ of material a radius $r$ from any vessel. Shown are $C F(r)$ for the images with the smallest and largest $C L$ $(\bullet C L=168 \pm 4 \mu \mathrm{m} ; \bullet C L=311 \pm 4 \mu \mathrm{m})$, with fitted curves. $C L$ is the distance (vertical reference line) marking the 18th percentile (horizontal reference line) of non-vascular tissue's proximity to a vessel. 
Table 1. Eight measures for eight images.

\begin{tabular}{|c|c|c|c|c|c|c|c|c|}
\hline Image & $V F_{O}$ & $D_{f}$ & $D_{f s k}$ & $C L(\mu \mathrm{m})$ & $C F_{0}=1-V F_{0}$ & $L_{q}(\mu \mathrm{m})$ & $L_{e}(\mu \mathrm{m})$ & $V F_{O}($ fitted $)$ \\
\hline CAMO & 0.17 & $1.48 \pm 0.03$ & $1.07 \pm 0.07$ & $226 \pm 8$ & 0.83 & $233 \pm 8$ & $158 \pm 9$ & $0.21 \pm 0.02$ \\
\hline CAM1 & 0.16 & $1.47 \pm 0.03$ & $1.05 \pm 0.07$ & $237 \pm 8$ & 0.83 & $256 \pm 25$ & $218 \pm 28$ & $0.19 \pm 0.03$ \\
\hline CAM2 & 0.15 & $1.43 \pm 0.02$ & $1.02 \pm 0.05$ & $239 \pm 5$ & 0.85 & $285 \pm 17$ & $245 \pm 12$ & $0.16 \pm 0.01$ \\
\hline CAM3 & 0.20 & $1.52 \pm 0.02$ & $1.04 \pm 0.06$ & $268 \pm 4$ & 0.80 & $405 \pm 8$ & $284 \pm 8$ & $0.22 \pm 0.02$ \\
\hline CAM4 & 0.23 & $1.54 \pm 0.04$ & $1.13 \pm 0.10$ & $168 \pm 4$ & 0.76 & $416 \pm 37$ & $255 \pm 15$ & $0.21 \pm 0.01$ \\
\hline CAM5 & 0.19 & $1.49 \pm 0.03$ & $1.09 \pm 0.08$ & $204 \pm 3$ & 0.80 & $264 \pm 13$ & $197 \pm 8$ & $0.21 \pm 0.02$ \\
\hline CAM7 & 0.20 & $1.53 \pm 0.02$ & $1.03 \pm 0.06$ & $311 \pm 4$ & 0.80 & $581 \pm 17$ & $421 \pm 16$ & $0.22 \pm 0.02$ \\
\hline
\end{tabular}

$V F_{O}$, vascular fraction, and $C F_{0}$, complement fraction, assumed to have no error. $V F_{0}$ (fitted) is usually larger than $V F_{0}$ from the raw image, because of a lower limit of capillary size. $D_{f}$, fractal dimension, and $D_{f s k}$, fractal dimension of the skeletonized image. $C L$, characteristic length, derived from shape of $C F(r)$. $L_{q}$ (quadratic) and $L_{e}$ (exponential), are lengths derived from shape of $V F(r)$. For definitions, see Appendix.

\subsection{Derived quantities}

Once we know the gauge-dependent vascular fraction $V F(r)$, the measure can be used to calculate derived measures for area density, length density and volume density at the different gauges. In the case of planar vasculature (such as in the CAM), the area, length and volume of vessels in a particular range of gauges $r_{1}$ to $r_{2}$ per unit area of tissue are given in table 2.

Hence, for example, the area density of vessels at all gauges is $-\int_{0}^{\infty}(\mathrm{d} / \mathrm{d} r)(V F(r)) \mathrm{d} r=V F(0)-V F(\infty)=$ $V F_{0}$, the vascular fraction. Also, we see that for planar vasculature, area density is equivalent to vascular fraction, since $A D(r, \infty)=-\int_{r}^{\infty}(\mathrm{d} / \mathrm{d} r)(V F(r)) \mathrm{d} r=V F(r)$.

Note that it is not possible to calculate the length density of vessels at the smallest gauges from the formula, because the formula for length density blows up at a radius $r_{1}=0$. The most widespread method of measuring length density, the grid intersection method, avoids this difficulty by missing many of the vessels smaller than the grid size. Real vessels, however, do have a minimum size, so the blowup of the length density at small radii presents little practical difficulty.

The volume density is well-behaved, and can be used as another measure of a vascular tree, if we are particularly concerned, for instance, with volumes of planar vascular trees, or with quantities closely related to volumes. Figure 9 illustrates the volume density distribution (integrand of volume density) for a CAM image whose $V F(r)$ was fitted by the quadratic and exponential functions $V F_{q}(r)$ and $V F_{e}(r)$.

Similarly, we can use $V F(r)$ measured from an image to derive any quantity which may depend on the radius of a vessel. For example, mean flow rate depends on the 4th power of the vessel radius, if Poiseuille's law applies.

Table 2

\begin{tabular}{lcc}
\hline Measure & \multicolumn{1}{c}{ units } & \multicolumn{1}{c}{ formula } \\
\hline Length density & length/area & $-\int_{r_{1}}^{r_{2}} \frac{1}{2 r} \frac{\mathrm{d}}{\mathrm{d} r}(V F(r)) \mathrm{d} r$ \\
$L D\left(r_{1}, r_{2}\right)$ & area/area & $-\int_{r_{1}}^{r_{2}} \frac{\mathrm{d}}{\mathrm{d} r}(V F(r)) \mathrm{d} r=V F\left(r_{1}\right)-V F\left(r_{2}\right)$ \\
Area density & & $-\int_{r_{1}}^{r_{2}} \frac{\pi r}{2} \frac{\mathrm{d}}{\mathrm{d} r}(V F(r)) \mathrm{d} r$ \\
$A D\left(r_{1}, r_{2}\right)$ & volume/area & \\
Volume density & & \\
$V D\left(r_{1}, r_{2}\right)$ & & \\
\hline
\end{tabular}

We can estimate the permeability coefficient $P$ as $P=$ $-\int_{0}^{\infty} r^{2}(\mathrm{~d} / \mathrm{d} r)(V F(r)) \mathrm{d} r$ such that the total flow rate through a vascular bed will be proportional to the product of $P$ and the ratio of pressure drop to viscosity [4]. There will be additional factors determining the actual flow rate, such as tortuosity and elasticity of the vessels, but our permeability coefficient $P$ gives a fair quantitative comparison between the flows expected in geometrically similar structures. $P$ has area units (length ${ }^{4}$ per unit area).

In figure 10 we see how the permeability is shared among vessels of different radii, and we see that the estimation of the permeability's dependence on radius also depends on which function is chosen to approximate $V F(r)$. The functions $V F_{q}(r)$ and $V F_{e}(r)$ are not fundamental to any measures, but are simply convenient ways of smoothing the $V F(r)$ data, which naturally have some roughness. Because of the simple form of $V F_{q}(r)$, we can estimate $P$ as the simple expression $\left(V F_{0} / 6\right) L_{q}^{2}$. The numerical estimate of $P$ by use of $V F_{e}(r)$ correlates very well ( $r=0.98$ ) with the estimate using $V F_{q}(r)$. Table 3 is a comparison of the permeability coefficients. Actual flow rate through a vascular bed would depend on vessel tortuosity and elasticity, the viscosity of the blood, and the pressure drop.

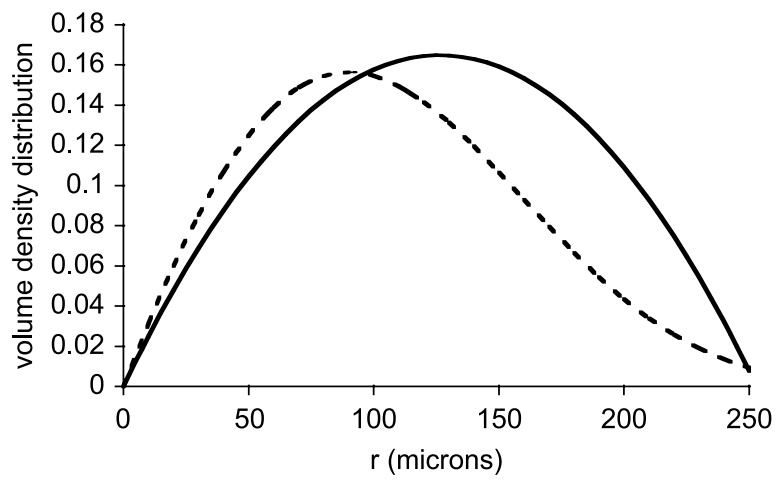

Figure 9. Planar volume density distribution (unitless) at a given gauge $r(\mu \mathrm{m})$, shown calculated from the fitted functions $V F_{q}(r)$ (solid curve) and $V F_{e}(r)$ (dashed curve) for the same image. Note that the greatest contribution to volume is at an intermediate gauge, and this gauge is somewhat different for the two functions. The total volume per unit area for this arterial tree is estimated differently by the exponential $\left(22.5 \mu \mathrm{m}^{3} / \mu \mathrm{m}^{2}\right)$ and quadratic $\left(27.8 \mu \mathrm{m}^{3} / \mu \mathrm{m}^{2}\right)$ functions. 


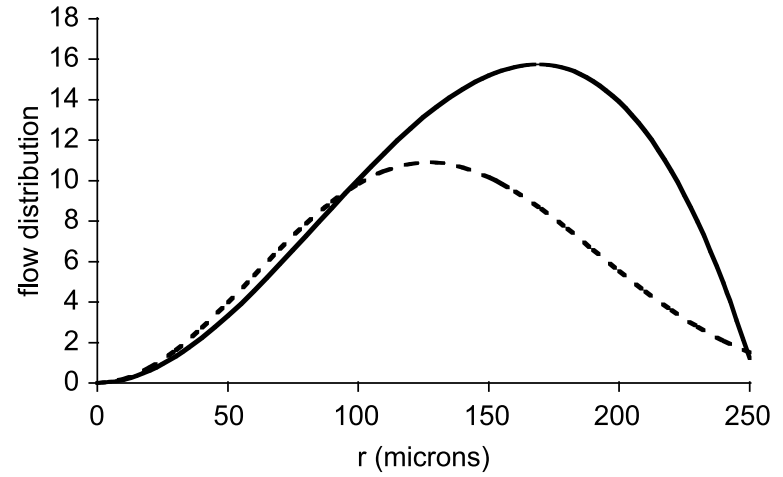

Figure 10. Distribution of flow capacity (arbitrary units) among vessels of gauge $r(\mu \mathrm{m})$, as estimated by two different functions $V F(r)$ for a particular CAM image. The quadratic function $V F_{q}(r)$ (solid curve) estimates a higher overall flow rate than the exponential function $V F_{e}(r)$ (dashed curve). Flow distribution units are arbitrary because actual flow rate depends on viscosity and pressure drop.

\subsection{Correlations}

Some of the measures we have examined for the CAM arterial trees are highly correlated, and others are uncorrelated (table 4). Interestingly, the two fractal dimensions $D_{f}$ and $D_{f s k}$ are only weakly correlated with each other. Fractal dimension $D_{f}$ is strongly correlated with $V F_{O}$, the fraction of the image which is vascular, and in fact the measured $V F_{O}$ correlates better with $D_{f}$ than it does with the fitted $V F_{O}$. On the other hand, the skeletonized fractal dimension $D_{f s k}$ is strongly negatively correlated with $C L$. In turn, $C L$ is uncorrelated with $D_{f}$ and with $V F_{O}$. Thus we could use $V F_{O}$ as a surrogate measure for $D_{f}$, and $C L$ as a surrogate measure for $D_{f s k} . L_{e}$ and $L_{q}$ are highly correlated, as is expected for different measures of the same feature, and they in turn are strongly correlated with the permeability coefficients $P_{e}$ and $P_{q}$. A principal component analysis (PCA) was performed, but the results do not provide new insight.

To distinguish one CAM arterial tree from another, in general it should suffice to report just three numbers. The measures should not be correlated with each other, or they will be presenting redundant information. A useful trio of independent, mostly uncorrelated measures could be $V F_{0}$, $C L$, and $P$. They represent, respectively, the fraction of tissue, which is vascular ( $V F_{0}$, a pure ratio), a measure of the distance of the vascularized tissue to its vessels $(C L$, a
Table 3. Estimates of permeability coefficient $P\left(\mu \mathrm{m}^{2}\right)$.

\begin{tabular}{lcr}
\hline Image & $P_{q}$ & $P_{e}$ \\
\hline CAM0 & 1900 & 1562 \\
CAM1 & 2075 & 2471 \\
CAM2 & 2166 & 2291 \\
CAM3 & 6014 & 5509 \\
CAM4 & 6057 & 4069 \\
CAM5 & 2439 & 2428 \\
CAM6 & 2306 & 2263 \\
CAM7 & 12377 & 12106 \\
\hline
\end{tabular}

$P$ is derived from vascular fraction $V F(r)$ of vessels of different radii, $P=-\int_{0}^{\infty} r^{2}(\mathrm{~d} / \mathrm{d} r)(V F(r)) \mathrm{d} r . P_{q}$ calculated from $V F_{q}(r)=V F_{0}\left(1-r / L_{q}\right)^{2}, P_{e}$ calculated from $V F_{e}(r)=\exp \left(\ln \left(V F_{0}\right) \exp \left(r / L_{e}\right)\right)$. True flow rate depends on several other factors, such as pressure drop and tortuosity.

length), and the flow capacity of the tissue ( $P$, an area). The three measures, along with fractal dimension $D_{f}$, are shown for several images in figure 11.

\section{Discussion}

The goal of this paper is to present a comparison of different common and uncommon measures of a vascular tree, with an eye to increasing the amount of biological insight gained from the use of these measures.

In particular, we find that the common fractal dimension $D_{f}$ has some common problems. First, the fractal dimension which is most widely reported as a vascular measure is actually of the skeletonized image, not the raw image, yet typically this distinction is not recognized. Second, it is not clear whether any groups reporting fractal dimensions of natural images have calibrated their algorithms on mathematical images of known $D_{f}$. When we did so with the most commonly used algorithm (gridbased box counting), we found a consistent underestimate of $D_{f}$, possibly due to prior compression of the fractal images by the authors who had generated them. We strongly recommend that researchers reporting fractal dimension of their natural images (a) indicate whether the images have been skeletonized, and (b) calibrate their algorithms on mathematical fractals.

We find that the abstract fractal dimension $D_{f}$ is really a surrogate measure for the vascular fraction $V F_{0}$. Since $V F_{0}$ is much more straightforward to measure, it would seem

Table 4. Correlations among measures reported in tables 1 and 2.

\begin{tabular}{|c|c|c|c|c|c|c|c|c|c|}
\hline & $V F_{O}$ meas & $V F_{O}$ fit & $C L$ & $L_{q}$ & $L_{e}$ & $D_{f}$ & $D_{f s k}$ & $P_{q}$ & $P_{e}$ \\
\hline$V F_{O}$ meas & 1.00 & & & & & & & & \\
\hline$V F_{0}$ fit & 0.72 & 1.00 & & & & & & & \\
\hline$C L$ & -0.24 & 0.14 & 1.00 & & & & & & \\
\hline$L_{q}$ & 0.65 & 0.49 & 0.46 & 1.00 & & & & & \\
\hline$L_{e}$ & 0.42 & 0.29 & 0.62 & 0.95 & 1.00 & & & & \\
\hline$D_{f}$ & 0.94 & 0.86 & 0.00 & 0.74 & 0.53 & 1.00 & & & \\
\hline$D_{f s k}$ & 0.64 & 0.36 & -0.84 & -0.09 & -0.34 & 0.48 & 1.00 & & \\
\hline$P_{q}$ & 0.62 & 0.54 & 0.52 & 0.99 & 0.95 & 0.74 & -0.12 & 1.00 & \\
\hline$P_{e}^{q}$ & 0.47 & 0.49 & 0.65 & 0.95 & 0.97 & 0.63 & -0.27 & 0.98 & 1.00 \\
\hline
\end{tabular}

Quantities which use different methods to measure the same physical feature (such as $L_{q}$ and $L_{e}$ ) should be highly correlated. Quantities which measure very different physical features should have low correlations, unless there is some biological reason for a high correlation. 


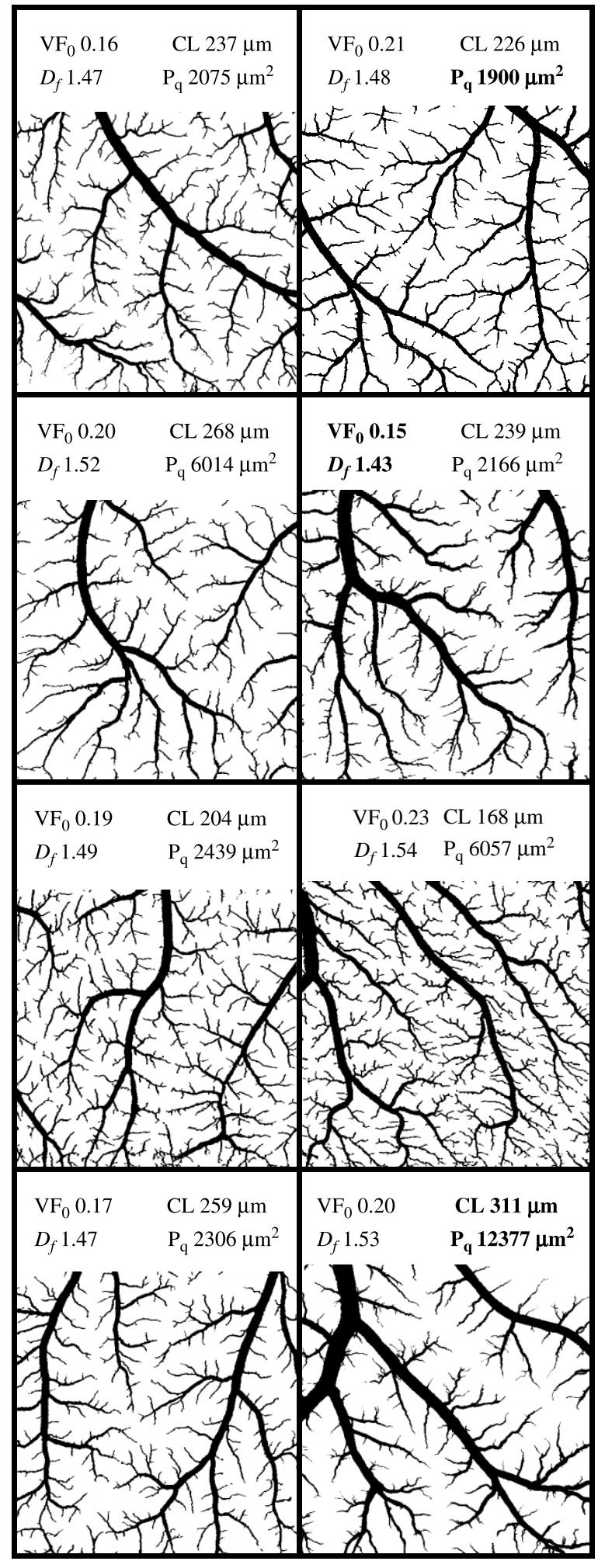

Figure 11. Eight images of CAM arterial trees. Trees on the left are in an intermediate range for the four measures shown. Trees on the right are at either a maximum or a minimum of one or more of the displayed measures (boldface).

superfluous to use $D_{f}$, which has no intrinsic biological meaning. More commonly used than $D_{f}$ is $D_{f s k}$, the fractal dimension of the skeletonized image. We have seen that (at least for the CAM) $D_{f s k}$ is a surrogate measure for $C L$, a length which is characteristic of the complement fraction $C F$, the space between the vessels. What is the $C L$ ? To be precise, it is the negative reciprocal of the initial slope of the curve of $C F(r) ; \exp (1-\exp (1))=18 \%$ of the nonvascular tissue, or $13-15 \%$ of the whole tissue, is farther from a vessel than that distance (as illustrated in figure 8). Therefore, the $C L$ is a measure of how close most of the interstitium is to the vessels, and it is a surrogate measure for $D_{f s k}$ (conversely, $D_{f s k}$ is a surrogate measure for how close most of the interstitium is to the vessels). The higher the fractal dimension $D_{f s k}$, the lower the characteristic length, and the closer most of the tissue is to the vessels. When we observe that the fractal dimension $D_{f s k}$ is increasing over time during development of the CAM, we can interpret it as the average piece of tissue getting closer to a vessel over time, the vasculature increasing its geometric efficiency.

A large number of derived measures based on the complement fraction $C F(r)$ are possible, and are straightforward to implement. For example, it is well known that tumor tissue has large avascular areas; this characteristic would appear quantitatively either as a large $C L$ or small $D_{f s k}$, or visually as the easily computed regions which are more than a particular distance from a vessel (figure 5).

Other authors [6,11] have found high correlations between $D_{f_{s k}}$ and what is called either $\rho_{\nu}$, vascular density, or $S_{i}$, vessel length. Note that $\rho_{\nu}$ is not the same as $V F_{0}$; it is the vascular fraction of the skeletonized image, hence is more correctly a measure of length density, or equivalent to $S_{i}$ per unit area. We may conclude that for CAM arterial trees, $D_{f s k}$ is a measure of both length density and of interstitial proximity, but is uncorrelated with any of the other measures we have examined.

The vascular measures based on digital "pruning" of the vascular tree allow us to estimate the effectiveness of functioning of the vascular tree with various derived measures. For example, we were able to use the measured functions $V F(r)$ to estimate the permeability coefficient $P$ of the vascular beds. If our primary biological interest is in studying the efficiency of a vascular tree and its dependence on our experimental conditions, the most important measurement of the tree may be $P$. If our interest is in determining whether a particular growth factor is affecting linear versus radial growth of the vasculature, the length density $L D\left(r_{1}, r_{2}\right)$ gives us a measure of the linear density of vessels in that particular range of gauges. $L D$ may be easier to implement than measures of branch generations [7,12].

We proposed a trio of independent, uncorrelated measures: $V F_{O}, C L$, and $P$. They represent, respectively, the fraction of tissue which is vascular $\left(V F_{0}\right.$, a pure ratio), the distance of the vascularized tissue to its vessels $(C L$, a length), and the flow capacity of the tissue ( $P$, an area). These units may be more intuitive than the fractal dimension, and two of the measures are highly correlated with fractal dimensions. Our proposed trio can replace fractal dimension as a measure of a vascular tree, and each 
Appendix: Measures discussed in this paper

\begin{tabular}{|c|c|c|c|}
\hline Symbol & Name & Units & Definition \\
\hline$D_{f}$ & fractal dimension & - & -slope of log-log plot of box counts at each gauge \\
\hline$D_{f s k}$ & fractal dimension of skeletonized image & - & - slope of $\log -\log$ plot of box counts at each gauge \\
\hline & gauge & length & pruning radius or dilation radius \\
\hline$V F(r)$ & volume fraction & - & $\begin{array}{l}\text { fraction of image consisting of vessels larger than a given } \\
\text { gauge } r\end{array}$ \\
\hline$C F(r)$ & complement fraction & - & $\begin{array}{l}\text { fraction of image which is farther from any vessel than } \\
\text { distance } r\end{array}$ \\
\hline$V F_{0}$ & volume fraction & - & fraction of image which is vascular tissue \\
\hline$C F_{0}$ & complement fraction & - & fraction of image which is not vascular tissue \\
\hline$C L$ & characteristic length of complement fraction & length & best-fitting parameter in $C F(r)=C F_{0} \exp \left(1-\exp \left(\frac{r}{C L}\right)\right)$ \\
\hline$V F_{q}(r)$ & quadratic fit of volume fraction & - & $V F_{q}(r)=V F_{0}\left(1-\frac{r}{L_{q}}\right)^{2}$ \\
\hline$V F_{e}(r)$ & compound exponential fit of volume fraction & - & $V F_{e}(r)=\exp \left(\ln \left(V F_{0}\right) \exp \left(\frac{r}{L_{e}}\right)\right)$ \\
\hline$L_{q}$ & characteristic length of vascular fraction & length & see definition for $V F_{q}(r)$; best fit to data \\
\hline$L_{e}$ & characteristic length of vascular fraction & length & see definition for $V F_{e}(r)$; best fit to data \\
\hline$L D\left(r_{1}, r_{2}\right)$ & length density & length/area & $-\int_{r_{1}}^{r_{2}} \frac{1}{2 r} \frac{\mathrm{d}}{\mathrm{d} r}(V F(r)) \mathrm{d} r$ \\
\hline$A D\left(r_{1}, r_{2}\right)$ & area density & area/area & $-\int_{r_{1}}^{r_{2}} \frac{\mathrm{d}}{\mathrm{d} r}(V F(r)) \mathrm{d} r=V F\left(r_{1}\right)-V F\left(r_{2}\right)$ \\
\hline$V D\left(r_{1}, r_{2}\right)$ & volume density & volume/area & $-\int_{r_{1}}^{r_{2}} \frac{\pi r}{2} \frac{\mathrm{d}}{\mathrm{d} r}(V F(r)) \mathrm{d} r$ \\
\hline$P$ & permeability coefficient & area & $P=-\int_{0}^{\infty} r^{2} \frac{\mathrm{d}}{\mathrm{d} r}(V F(r)) \mathrm{d} r$ \\
\hline$P_{q}$ & permeability coefficient (quadratic method) & area & $P_{q}=-\int_{0}^{\infty} r^{2} \frac{\mathrm{d}}{\mathrm{d} r}\left(V F_{q}(r)\right) \mathrm{d} r=\frac{V F_{0}}{6} L_{q}^{2}$ \\
\hline$P_{e}$ & permeability coefficient (compound exponential method) & area & $P_{e}=-\int_{0}^{\infty} r^{2} \frac{\mathrm{d}}{\mathrm{d} r}\left(V F_{e}(r)\right) \mathrm{d} r$ \\
\hline
\end{tabular}

of our three measures has a straightforward biological interpretation.

\section{Acknowledgements}

This work has been partially supported by grants NSF(-NIH) DMS-NIGMS 0201094 (SRL), NSF EEC9529161 (SF, EHS), and NIH R01-GM40711 (SF, EHS).

\section{References}

[1] Baish, J.W. and Jain, R.K., 2000, Fractals and cancer. Cancer Res. 60, 3683-3688.

[2] Bassingthwaighte, J.B., Liebovitch, L.S. and West, B.J., 1994, Fractal Physiology (Oxford: Oxford University Press).

[3] Chantrain, C.F., DeClerck, Y.A., Groshen, S. and McNamara, G., 2003, Computerized quantification of tissue vascularization using high-resolution slide scanning of whole tumor sections. J. Histochem Cytochem. 51(2), 151-158.

[4] Childs, E.C. and Collis-George, N., 1950, Permeability of porous materials. Proc. Roy. Soc. Lond. A. 201(1066), 392-405.

[5] Kirchner, L.M., Schmidt, S.P. and Gruber, B.S., 1996, Quantitation of angiogenesis in the chick chorioallantoic membrane model using fractal analysis. Microvasc. Res. 51, 2-14.
[6] Parsons-Wingerter, P, et al., 1998, A novel assay of angiogenesis in the quail chorioallantoic membrane: stimulation by bFGF and inhibition by angiostatin according to fractal dimension and grid intersection. Microvasc. Res. 55, 201-214.

[7] Parsons-Wingerter, P., Elliott, K.E., Farr, A.G., Radhakrishnan, K., Clark, J.I. and Sage, E.H., 2000, Generational analysis reveals that TGF-beta 1 inhibits the rate of angiogenesis in vivo by selective decrease in the number of new vessels. Microvasc. Res. 59(2), 221-232.

[8] Sabo, E., Boltenko, A., Sova, Y., Stein, A., Kleinhaus, S. and Resnick, M.B., 2001, Microscopic analysis and significance of vascular architectural complexity in renal cell carcinoma. Clin. Cancer. Res. 7, 533-537.

[9] Thompson, W.D. and Reid, A., 2000, Quantitative assays for the chick chorioallantoic membrane. Angiogenesis: From the Molecular to Integrative Pharmacology, 476, 225-236.

[10] Wild, R., Ramakrishnan, S., Sedgewick, J. and Griffioen, A.W., 2000, Quantitative assessment of angiogenesis and tumor vessel architecture by computer-assisted digital image analysis: Effects of VEGF-toxin conjugate on tumor microvessel density. Microvasc. Res. 59(3), 368-376.

[11] Vico, P.G., Kyriacos, S., Heymans, O., Louryan, S. and Cartilier, L., 1998, Dynamic study of the extraembryonic vascular network of the chick embryo by fractal analysis. J. Theor. Biol. 195(4), 525-532.

[12] Zamir, M., 1997, On fractal properties of arterial trees. J. Theor. Biol. 197(4), 517-526.

[13] Zamir, M., 2001, Fractal dimensions and multifractility in vascular branching. J. Theor. Biol. 212(2), 183-190. 


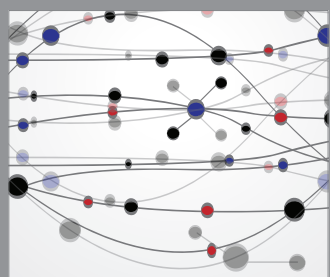

The Scientific World Journal
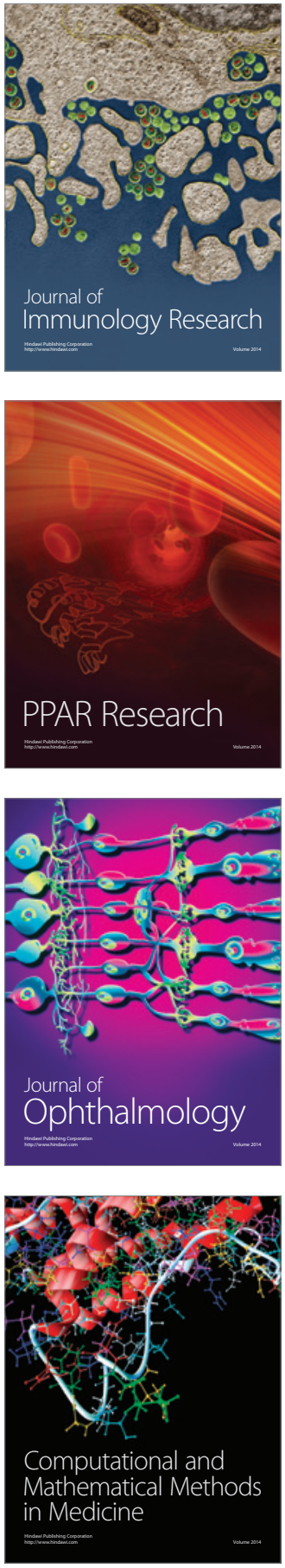

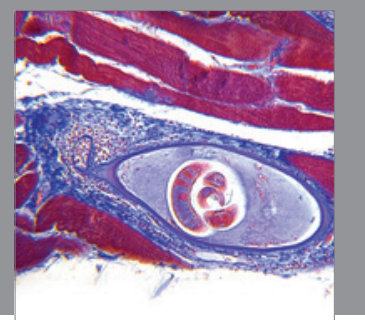

Gastroenterology

Research and Practice
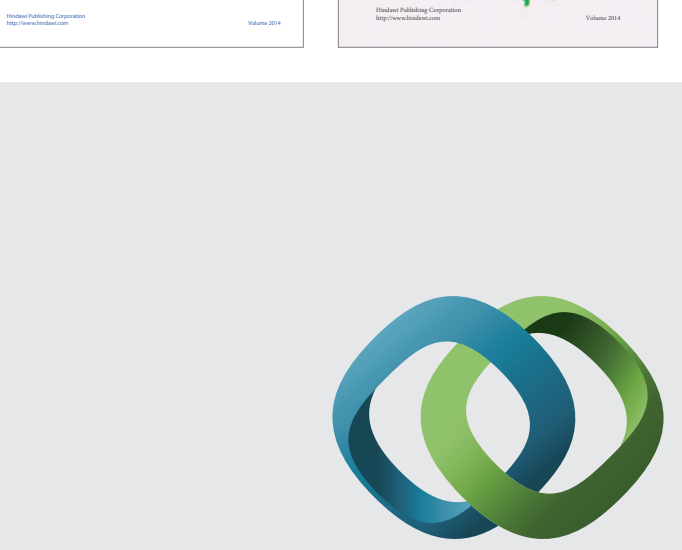

\section{Hindawi}

Submit your manuscripts at

http://www.hindawi.com
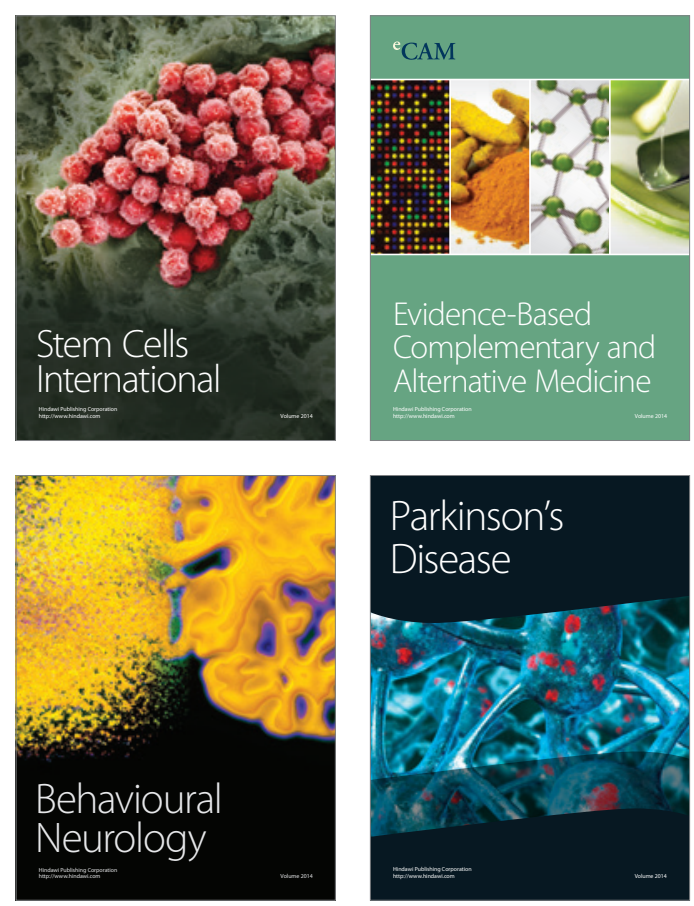

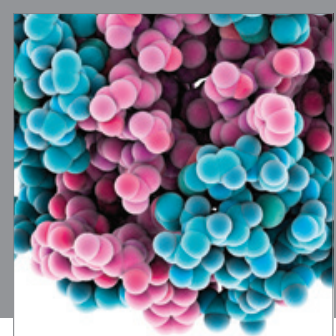

Journal of
Diabetes Research

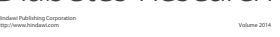

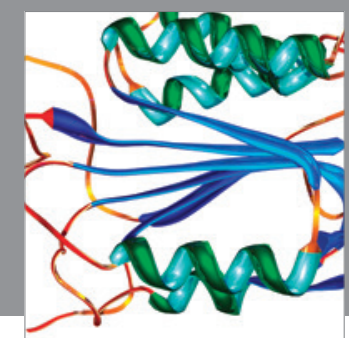

Disease Markers
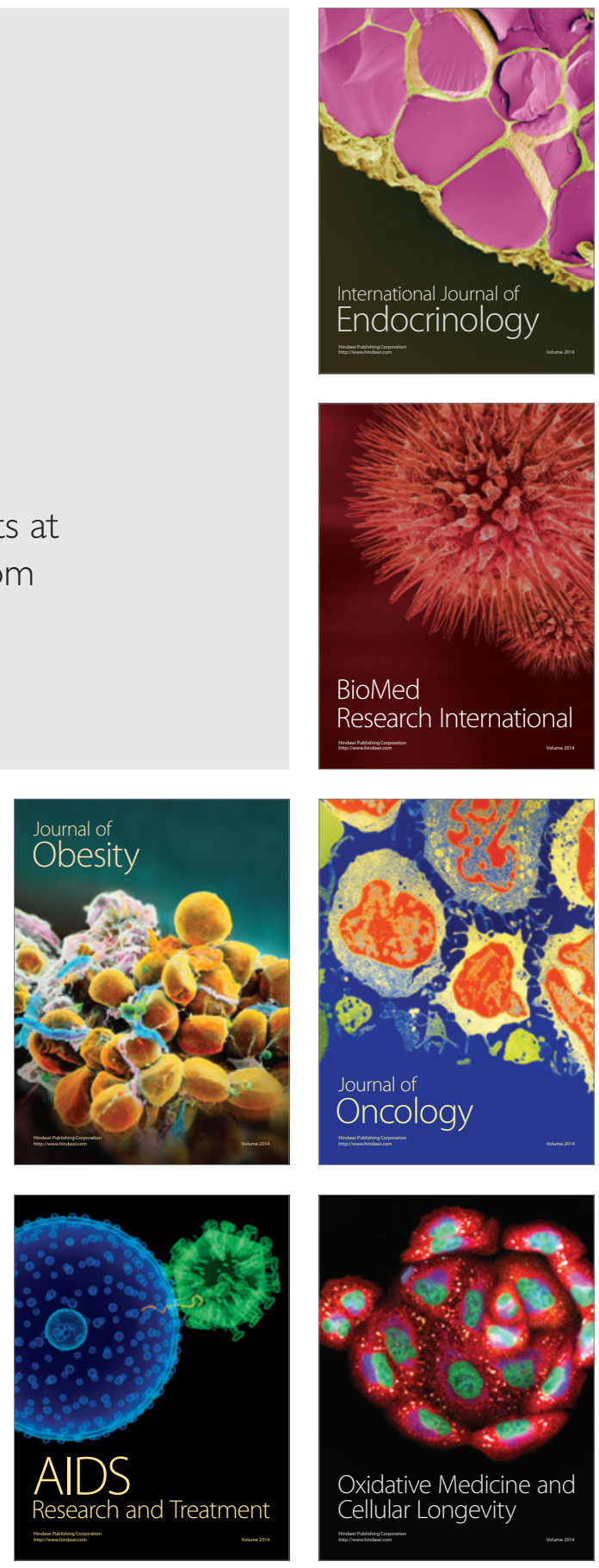